\title{
Metric properties for $p$-adic Oppenheim series expansions
}

\author{
by \\ Jun Wu (Wuhan)
}

1. Introduction. Real numbers have several representations, such as continued fraction expansions, Lüroth series, Engel series, Sylvester series expansions and Cantor infinite products etc. (see [4] and [20]). Analogous to continued fraction expansions, certain types of $p$-adic continued fractions have been studied by many mathematicians; see for example, [15], [17], [13] and [14] etc. In [8]-[10], A. Knopfmacher and J. Knopfmacher introduced and studied some properties of various unique $p$-adic expansions as sums of reciprocals of $p$-adic numbers with $p$-adic valuations not less than 1 . These expansions, including $p$-adic Lüroth series, Engel series, Sylvester series expansions and $p$-adic Cantor infinite products, were constructed to be analogous to the so-called Oppenheim series expansions of real numbers discussed in Galambos [4].

In the direction of metric and asymptotic results concerning digits, various results were established; in particular, for expansions of real numbers, by Jager and de Vroedt [6] and Salát [18] for real Lüroth series expansions, Erdős, Rényi and Szüsz [2] for real Engel and Sylvester series expansions, Rényi [16] for real Cantor infinite products and by Galambos [4] for more general situations, called Oppenheim series expansions of real numbers. Ruban [17] established $p$-adic metric theorems analogous to some of Khinchin [7] for real continued fractions. The corresponding results for $p$-adic Lüroth and Engel series expansions have been derived by A. Knopfmacher and J. Knopfmacher [11] and Grabner and A. Knopfmacher [5], respectively.

The main aim of this paper is to derive metric and asymptotic results for $p$-adic Oppenheim series expansions. We generalize the results obtained by A. Knopfmacher and J. Knopfmacher [11] and Grabner and A. Knopfmacher [5] for $p$-adic Lüroth and Engel series expansions. Also as special

2000 Mathematics Subject Classification: Primary 11K55, 11K41; Secondary 28A80.

This work is supported by the Kua-Shi-Ji foundation of Educational Committee and the Special Funds for Major State Basic Research Projects of China. 
cases of our results, we give metric results for $p$-adic Sylvester series expansions and $p$-adic Cantor infinite products. The corresponding results for Oppenheim series expansions of Laurent series have been obtained by Fan and the author [3].

2. The $p$-adic Oppenheim series expansions. In order to explain the conclusions, we first fix some notations and describe the $p$-adic Oppenheim series expansions to be considered.

Let us give a brief account of $p$-adic numbers; more details can be found in the books by Koblitz [12] and Schikhof [19].

Let $p$ be a fixed prime number. Every non-zero rational number $A$ can be expressed uniquely in the form $A=p^{a} r / s$, where $(r, p)=(s, p)=1$ and $a \in \mathbb{Z}$. The $p$-adic valuation ||$_{p}$ on $\mathbb{Q}$ is defined to be

$$
|A|_{p}=p^{-a} \quad \text { if } A \neq 0, \quad|0|_{p}=0 .
$$

The completion of $\mathbb{Q}$ with respect to the $p$-adic metric ||$_{p}$ gives rise to the field $\mathbb{Q}_{p}$. Each element $A \in \mathbb{Q}_{p}$ has a unique series representation

$$
A=\sum_{n=m}^{\infty} c_{n} p^{n},
$$

where $m \in \mathbb{Z}$ and the coefficients $c_{n}$ are rational integers satisfying $0 \leq c_{n}$ $\leq p-1$ and $c_{m} \neq 0$. The integer $m$ is called the order of $A$ and denoted by $v(A)$, and $|A|_{p}=p^{-m}$. The valuation ||$_{p}$ defined on $\mathbb{Q}_{p}$ has the properties

$$
\begin{aligned}
& |A|_{p} \geq 0, \quad|A|_{p}=0 \text { if and only if } A=0, \quad|A B|_{p}=|A|_{p}|B|_{p}, \\
& |A+B|_{p} \leq \max \left(|A|_{p},|B|_{p}\right) \quad \text { with equality when }|A|_{p} \neq|B|_{p} .
\end{aligned}
$$

For $v(A)$, we have

$$
\begin{gathered}
v(0)=\infty, \quad v(A B)=v(A)+v(B), \quad v(A / B)=v(A)-v(B) \quad \text { if } B \neq 0, \\
v(A+B) \geq \min (v(A), v(B)) \quad \text { with equality when } v(A) \neq v(B) .
\end{gathered}
$$

It is well known that the above non-Archimedean valuation leads to an ultrametric distance function $\varrho$, with $\varrho(A, B)=|A-B|_{p}$, making $\mathbb{Q}_{p}$ into a complete metric space with respect to $\varrho$.

REMARK 2.1. Since the metric $\varrho$ is non-Archimedean, it follows that each point of a disc may be considered its center and thus if two discs intersect, then one contains the other.

For any $A \in \mathbb{Q}_{p}$, if $A=\sum_{n=v(A)}^{\infty} c_{n} p^{n}$, we call the finite series $\langle A\rangle=$ $\sum_{v(A) \leq n \leq 0} c_{n} p^{n}$ the fractional part of $A$. Then $\langle A\rangle \in S_{p}$, where we define $S_{p}=\left\{\langle A\rangle: A \in \mathbb{Q}_{p}\right\} \subset \mathbb{Q}$. The set $S_{p}$ is multiplicatively but not additively closed. The function $\langle A\rangle$ and set $S_{p}$ have been used in the study of 
certain types of $p$-adic continued fractions by Mahler [15], Ruban [17] and Laohakosol [13] in particular.

For any $n \geq 1$, let $r_{n}, s_{n}$ be maps from $p^{-1}\left(S_{p} \backslash\{0\}\right)$ to $\mathbb{Q} \backslash\{0\}$ satisfying, for any $a \in p^{-1}\left(S_{p} \backslash\{0\}\right)$,

$$
\begin{gathered}
2 v(a)-v\left(s_{n}(a)\right)+v\left(r_{n}(a)\right) \leq 0 \quad \text { for any } n \geq 1, \\
v\left(r_{n}(a)\right)=v\left(r_{n}\left(a^{\prime}\right)\right), \quad v\left(s_{n}(a)\right)=v\left(s_{n}\left(a^{\prime}\right)\right) \quad \text { if } v(a)=v\left(a^{\prime}\right) .
\end{gathered}
$$

Given any $A \in \mathbb{Q}_{p}$, note that $\langle A\rangle=a_{0} \in S_{p}$ if and only if $v\left(A-a_{0}\right) \geq 1$. Then define $A_{1}=A-a_{0}$. As in [9], [10], if $A_{n} \neq 0$ with $v\left(A_{n}\right) \geq 1(n \geq 1)$ is already defined, then define the "digit" $a_{n}=\left\langle 1 / A_{n}\right\rangle$ and put

$$
A_{n+1}=\left(A_{n}-\frac{1}{a_{n}}\right) \frac{s_{n}\left(a_{n}\right)}{r_{n}\left(a_{n}\right)} .
$$

For any $m \geq 1$, if $A_{m} \neq 0$, by (1) and [10, (2.3)], we have $v\left(A_{m}\right) \geq 1$. If some $A_{m}=0$, this recursive process stops. It was shown in [9], [10] that this algorithm leads to a finite or convergent series (relative to $\varrho$ ), called the p-adic Oppenheim series expansion.

Theorem $2.2([9],[10])$. Every $x \in \mathbb{Q}_{p}$ has a finite or convergent (relative to $\varrho$ ) series expansion of the form

$$
x=a_{0}(x)+\frac{1}{a_{1}(x)}+\sum_{n=1}^{\infty} \frac{r_{1}\left(a_{1}(x)\right) \ldots r_{n}\left(a_{n}(x)\right)}{s_{1}\left(a_{1}(x)\right) \ldots s_{n}\left(a_{n}(x)\right)} \frac{1}{a_{n+1}(x)},
$$

where $a_{n}(x) \in S_{p}, a_{0}(x)=\langle x\rangle$, and $v\left(a_{1}(x)\right) \leq 1$, for any $n \geq 1$,

$$
v\left(a_{n+1}(x)\right) \leq 2 v\left(a_{n}(x)\right)-1+v\left(r_{n}\left(a_{n}(x)\right)\right)-v\left(s_{n}\left(a_{n}(x)\right)\right) .
$$

The expansion is unique for $x$ subject to the above conditions on the "digits" $a_{n}(x)$.

REMARK 2.3. The algorithm above is more restricted than the general algorithm described in [10] in order to obtain our metric results. (1) is used to guarantee that $v\left(a_{n}\right) \leq-1$ for any $n \geq 1$ if the process does not stop (see (5)).

Here are some special cases:

p-adic Lüroth series expansion: $s_{n}(a)=a(a-1), r_{n}(a)=1$;

p-adic Engel expansion: $s_{n}(a)=a, r_{n}(a)=1$;

p-adic Sylvester expansion: $s_{n}(a)=1, r_{n}(a)=1$;

p-adic Cantor infinite product: $s_{n}(a)=a, r_{n}(a)=a+1$.

Let $X_{p}=p \mathbb{Z}_{p}$ denote the maximal ideal in the ring $\mathbb{Z}_{p}$ of all $p$-adic integers, i.e. the set of $p$-adic numbers of order $\geq 0$. Then $X_{p}$ is compact. For any $A \in X_{p}, v(A) \geq 1$ and from Remark 2.3,v( $\left.A_{n}\right) \geq 1$ for any $n \geq 1$ if the process does not stop. Let $\mathbf{P}$ be the probability measure with respect to 
Haar measure on $\mathbb{Q}_{p}$ normalized by $\mathbf{P}\left(X_{p}\right)=1$. A convenient description of $\mathbf{P}$ on $X_{p}$ is given in Sprindžuk [21, pp. 67-70]. In particular, $\mathbf{P}(C)=p^{-m}$ for any disc

$$
C=C\left(x, p^{-m-1}\right):=\left\{y \in \mathbb{Q}_{p}:|y-x|_{p} \leq p^{-m-1}\right\}
$$

of radius $p^{-m-1}$.

For any $x \in X_{p}$, let $\left\{\triangle_{n}(x): n \geq 0\right\}$ denote the sequence of random variables such that $\triangle_{0}(x)=v\left(a_{1}(x)\right), \triangle_{n}(x)=v\left(a_{n+1}(x)\right)-2 v\left(a_{n}(x)\right)-$ $v\left(r_{n}\left(a_{n}(x)\right)\right)+v\left(s_{n}\left(a_{n}(x)\right)\right)$ for $n \geq 1$.

Now we state our main results.

Theorem 2.4. For the p-adic Oppenheim series expansions described above:

(i) $\lim _{n \rightarrow \infty} \mathbf{P}\left\{x \in X_{p}: \frac{\sum_{j=0}^{n-1} \triangle_{j}(x)+\frac{p}{p-1} n}{\sqrt{n p} /(p-1)}<t\right\}=\frac{1}{\sqrt{2 \pi}} \int_{-\infty}^{t} e^{-u^{2} / 2} d u$.

(ii) For $\mathbf{P}$-almost all $x \in X_{p}$,

$$
\lim _{n \rightarrow \infty} \frac{1}{n} \sum_{j=0}^{n-1} \triangle_{j}(x)=-\frac{p}{p-1}
$$

(iii) For $\mathbf{P}$-almost all $x \in X_{p}$,

$$
\begin{aligned}
& \limsup _{n \rightarrow \infty} \frac{\sum_{j=0}^{n-1} \triangle_{j}(x)+\frac{p}{p-1} n}{\sqrt{2 n \log \log n}}=\frac{\sqrt{p}}{p-1}, \\
& \liminf _{n \rightarrow \infty} \frac{\sum_{j=0}^{n-1} \triangle_{j}(x)+\frac{p}{p-1} n}{\sqrt{2 n \log \log n}}=-\frac{\sqrt{p}}{p-1} .
\end{aligned}
$$

Furthermore, we consider the random variables

$$
\left|\frac{a_{n+1}(x) s_{n}\left(a_{n}(x)\right)}{a_{n}(x)^{2} r_{n}\left(a_{n}(x)\right)}\right|_{p}=p^{-\triangle_{n}(x)}, \quad n=1,2, \ldots
$$

In Proposition 3.5, we will show that these are independent and identically distributed with infinite expectation. However, we have the following result:

Theorem 2.5. For any fixed $\varepsilon>0$,

$$
\lim _{n \rightarrow \infty} \mathbf{P}\left\{x \in X_{p}:\left.\left|\frac{1}{n \log _{p} n} \sum_{j=1}^{n}\right| \frac{a_{j+1}(x) s_{j}\left(a_{j}(x)\right)}{a_{j}(x)^{2} r_{j}\left(a_{j}(x)\right)}\right|_{p}-(p-1) \mid>\varepsilon\right\}=0,
$$

i.e.

$$
\frac{1}{n \log _{p} n} \sum_{j=1}^{n}\left|\frac{a_{j+1}(x) s_{j}\left(a_{j}(x)\right)}{a_{j}(x)^{2} r_{j}\left(a_{j}(x)\right)}\right|_{p} \rightarrow p-1 \quad \text { in probability. }
$$

This paper is organized as follows. In Section 3, we give the proof of Theorem 2.4. Section 4 is devoted to the proof of Theorem 2.5. 
3. Proof of Theorem 2.4. In order to prove Theorem 2.4, we need some preliminary results.

Lemma 3.1. For any $k_{1}, \ldots, k_{n} \in S_{p}$ satisfying $v\left(k_{1}\right) \leq-1, \quad v\left(k_{j+1}\right) \leq 2 v\left(k_{j}\right)-1+v\left(r_{j}\left(k_{j}\right)\right)-v\left(s_{j}\left(k_{j}\right)\right), \quad 1 \leq j \leq n-1$, we have $\mathbf{P}\left\{x \in X_{p}: a_{1}(x)=k_{1}, \ldots, a_{n}(x)=k_{n}\right\}=p^{-\sum_{j=1}^{n-1}\left(v\left(r_{j}\left(k_{j}\right)\right)-v\left(s_{j}\left(k_{j}\right)\right)\right)+2 v\left(k_{n}\right)}$.

Proof. From (5), we have

$$
v\left(\frac{r_{1}\left(k_{1}\right) \ldots r_{n}\left(k_{n}\right)}{s_{1}\left(k_{1}\right) \ldots s_{n}\left(k_{n}\right)} \frac{1}{a_{n+1}(x)}\right) \geq v\left(\frac{r_{1}\left(k_{1}\right) \ldots r_{n-1}\left(k_{n-1}\right)}{s_{1}\left(k_{1}\right) \ldots s_{n-1}\left(k_{n-1}\right)} \frac{1}{a_{n}(x)}\right)+1 .
$$

Thus by Theorem 2.2, $\left\{x \in X_{p}: a_{1}(x)=k_{1}, \ldots, a_{n}(x)=k_{n}\right\}$ is a disc with center at

$$
\frac{1}{k_{1}}+\sum_{j=2}^{n} \frac{r_{1}\left(k_{1}\right) \ldots r_{j-1}\left(k_{j-1}\right)}{s_{1}\left(k_{1}\right) \ldots s_{j-1}\left(k_{j-1}\right)} \frac{1}{k_{j}}
$$

and diameter

$$
p^{-\sum_{j=1}^{n-1}\left(v\left(r_{j}\left(k_{j}\right)\right)-v\left(s_{j}\left(k_{j}\right)\right)\right)+2 v\left(k_{n}\right)-1} .
$$

Thus

$$
\mathbf{P}\left\{x \in X_{p}: a_{1}(x)=k_{1}, \ldots, a_{n}(x)=k_{n}\right\}=p^{-\sum_{j=1}^{n-1}\left(v\left(r_{j}\left(k_{j}\right)\right)-v\left(s_{j}\left(k_{j}\right)\right)\right)+2 v\left(k_{n}\right)} .
$$

Proposition 3.2. For any $k_{1}, \ldots, k_{n+1} \in S_{p}$ satisfying

$$
v\left(k_{1}\right) \leq-1, \quad v\left(k_{j+1}\right) \leq 2 v\left(k_{j}\right)-1+v\left(r_{j}\left(k_{j}\right)\right)-v\left(s_{j}\left(k_{j}\right)\right), \quad 1 \leq j \leq n,
$$

we have

$$
\begin{aligned}
\mathbf{P}\left\{a_{n+1}(x)=k_{n+1}\right. & \left.\mid a_{n}(x)=k_{n}\right\} \\
& =\mathbf{P}\left\{a_{n+1}(x)=k_{n+1} \mid a_{1}(x)=k_{1}, \ldots, a_{n}(x)=k_{n}\right\} \\
& =\left|\frac{r_{n}\left(k_{n}\right)}{s_{n}\left(k_{n}\right)}\right|_{p} \frac{\left|k_{n}\right|_{p}^{2}}{\left|k_{n+1}\right|_{p}^{2}},
\end{aligned}
$$

i.e. $\left\{a_{n}(x): n \geq 1\right\}$ forms a Markov chain with transition probabilities,

$$
\mathbf{P}\left\{a_{n+1}(x)=l_{n+1} \mid a_{n}(x)=l_{n}\right\}=\left|\frac{r_{n}\left(l_{n}\right)}{s_{n}\left(l_{n}\right)}\right|_{p} \frac{\left|l_{n}\right|_{p}^{2}}{\left|l_{n+1}\right|_{p}^{2}}
$$

if $v\left(l_{n+1}\right) \leq 2 v\left(l_{n}\right)-1+v\left(r_{n}\left(l_{n}\right)\right)-v\left(s_{n}\left(l_{n}\right)\right)$, and 0 otherwise.

Proof. By Lemma 3.1,

$$
\begin{aligned}
\mathbf{P}\left\{a_{n+1}(x)\right. & \left.=k_{n+1} \mid a_{n}(x)=k_{n}, \ldots, a_{1}(x)=k_{1}\right\} \\
& =\frac{\mathbf{P}\left\{a_{1}(x)=k_{1}, \ldots, a_{n}(x)=k_{n}, a_{n+1}(x)=k_{n+1}\right\}}{\mathbf{P}\left\{a_{1}(x)=k_{1}, \ldots, a_{n-1}(x)=k_{n-1}, a_{n}(x)=k_{n}\right\}} \\
& =\frac{p^{-\sum_{j=1}^{n}\left(v\left(r_{j}\left(k_{j}\right)\right)-v\left(s_{j}\left(k_{j}\right)\right)\right)+2 v\left(k_{n+1}\right)}}{p^{-\sum_{j=1}^{n-1}\left(v\left(r_{j}\left(k_{j}\right)\right)-v\left(s_{j}\left(k_{j}\right)\right)\right)+2 v\left(k_{n}\right)}}=\left|\frac{r_{n}\left(k_{n}\right)}{s_{n}\left(k_{n}\right)}\right|_{p} \frac{\left|k_{n}\right|_{p}^{2}}{\left|k_{n+1}\right|_{p}^{2}} .
\end{aligned}
$$


On the other hand,

$$
\begin{aligned}
\mathbf{P}\{ & \left.a_{n+1}(x)=k_{n+1} \mid a_{n}(x)=k_{n}\right\} \\
& =\frac{\mathbf{P}\left\{a_{n}(x)=k_{n}, a_{n+1}(x)=k_{n+1}\right\}}{\mathbf{P}\left\{a_{n}(x)=k_{n}\right\}} \\
& =\frac{\sum \mathbf{P}\left\{a_{j}(x)=l_{j}, 1 \leq j \leq n-1, a_{n}(x)=k_{n}, a_{n+1}(x)=k_{n+1}\right\}}{\sum \mathbf{P}\left\{a_{j}(x)=m_{j}, 1 \leq j \leq n-1, a_{n}(x)=k_{n}\right\}} \\
& =\frac{\sum p^{-\sum_{j=1}^{n-1}\left(v\left(r_{j}\left(l_{j}\right)\right)-v\left(s_{j}\left(l_{j}\right)\right)-\left(v\left(r_{n}\left(k_{n}\right)\right)-v\left(s_{n}\left(k_{n}\right)\right)\right)+2 v\left(k_{n+1}\right)\right.}}{\sum p^{-\sum_{j=1}^{n-1}\left(v\left(r_{j}\left(m_{j}\right)\right)-v\left(s_{j}\left(m_{j}\right)\right)\right)+2 v\left(k_{n}\right)}} \\
= & \left|\frac{r_{n}\left(k_{n}\right)}{s_{n}\left(k_{n}\right)}\right|_{p} \frac{\left|k_{n}\right|_{p}^{2}}{\left|k_{n+1}\right|_{p}^{2}},
\end{aligned}
$$

where the summations in the numerators of (6) and (7) are over all $l_{1}, \ldots, l_{n-1} \in S_{p}$ satisfying $v\left(l_{1}\right) \leq-1, v\left(l_{j+1}\right) \leq 2 v\left(l_{j}\right)-1+v\left(r_{j}\left(l_{j}\right)\right)-$ $v\left(s_{j}\left(l_{j}\right)\right)$ for $1 \leq j \leq n-2$ and $v\left(k_{n}\right) \leq 2 v\left(l_{n-1}\right)-1+v\left(r_{n-1}\left(l_{n-1}\right)\right)-$ $v\left(s_{n-1}\left(l_{n-1}\right)\right)$, and the summations in the denominators of (6) and (7) are over all $m_{1}, \ldots, m_{n-1} \in S_{p}$ satisfying $v\left(m_{1}\right) \leq-1, v\left(m_{j+1}\right) \leq 2 v\left(m_{j}\right)-$ $1+v\left(r_{j}\left(m_{j}\right)\right)-v\left(s_{j}\left(m_{j}\right)\right)$ for $1 \leq j \leq n-2$ and $v\left(k_{n}\right) \leq 2 v\left(m_{n-1}\right)-1+$ $v\left(r_{n-1}\left(m_{n-1}\right)\right)-v\left(s_{n-1}\left(m_{n-1}\right)\right)$.

Next we show that $\left\{v\left(a_{n}(x)\right): n \geq 1\right\}$ forms a Markov chain.

Lemma 3.3. For any $k_{1}, \ldots, k_{n} \in S_{p}$ as in Lemma 3.1, we have

$$
\begin{aligned}
\mathbf{P}\left\{x \in X_{p}: v\left(a_{1}(x)\right)=v\left(k_{1}\right), \ldots, v\left(a_{n}(x)\right)=v\left(k_{n}\right)\right\} \\
=(p-1)^{n} p^{-\sum_{j=1}^{n-1} v\left(k_{j}\right)+v\left(k_{n}\right)} p^{-\sum_{j=1}^{n-1}\left(v\left(r_{j}\left(k_{j}\right)\right)-v\left(s_{j}\left(k_{j}\right)\right)\right)} .
\end{aligned}
$$

Proof. By Lemma 3.1 and (2),

$$
\mathbf{P}\left\{x \in X_{p}: v\left(a_{1}(x)\right)=v\left(k_{1}\right), \ldots, v\left(a_{n}(x)\right)=v\left(k_{n}\right)\right\}
$$

$$
\begin{aligned}
& =\sum \mathbf{P}\left\{a_{1}(x)=l_{1}, \ldots, a_{n}(x)=l_{n}\right\} \\
& =\sum p^{-\sum_{j=1}^{n-1}\left(v\left(r_{j}\left(l_{j}\right)\right)-v\left(s_{j}\left(l_{j}\right)\right)\right)+2 v\left(l_{n}\right)} \\
& =\sum p^{-\sum_{j=1}^{n-1}\left(v\left(r_{j}\left(k_{j}\right)\right)-v\left(s_{j}\left(k_{j}\right)\right)\right)+2 v\left(k_{n}\right)} \\
& =(p-1)^{n} p^{-\sum_{j=1}^{n} v\left(k_{j}\right)+2 v\left(k_{n}\right)} p^{-\sum_{j=1}^{n-1}\left(v\left(r_{j}\left(k_{j}\right)\right)-v\left(s_{j}\left(k_{j}\right)\right)\right)} \\
& =(p-1)^{n} p^{-\sum_{j=1}^{n-1} v\left(k_{j}\right)+v\left(k_{n}\right)} p^{-\sum_{j=1}^{n-1}\left(v\left(r_{j}\left(k_{j}\right)\right)-v\left(s_{j}\left(k_{j}\right)\right)\right)},
\end{aligned}
$$

where the summations in (8), (9) and (10) are over all $l_{1}, \ldots, l_{n} \in S_{p}$ such that $v\left(l_{j}\right)=v\left(k_{j}\right), 1 \leq j \leq n$. 
Proposition 3.4. For any $k_{1}, \ldots, k_{n+1} \in S_{p}$ as in Proposition 3.2, we have

$$
\begin{aligned}
& \mathbf{P}\left\{v\left(a_{n+1}(x)\right)=v\left(k_{n+1}\right) \mid v\left(a_{n}(x)\right)=v\left(k_{n}\right)\right\} \\
& \quad=\mathbf{P}\left\{v\left(a_{n+1}(x)\right)=v\left(k_{n+1}\right) \mid v\left(a_{1}(x)\right)=v\left(k_{1}\right), \ldots, v\left(a_{n}(x)\right)=v\left(k_{n}\right)\right\} \\
& \quad=(p-1) p^{v\left(k_{n+1}\right)-2 v\left(k_{n}\right)-v\left(r_{n}\left(k_{n}\right)\right)+v\left(s_{n}\left(k_{n}\right)\right)} .
\end{aligned}
$$

Proof. By Lemma 3.3,

$$
\begin{aligned}
\mathbf{P}\left\{v\left(a_{n+1}(x)\right)=\right. & \left.v\left(k_{n+1}\right) \mid v\left(a_{n}(x)\right)=v\left(k_{n}\right), \ldots, v\left(a_{1}(x)\right)=v\left(k_{1}\right)\right\} \\
= & \frac{\mathbf{P}\left\{v\left(a_{1}(x)\right)=v\left(k_{1}\right), \ldots, v\left(a_{n+1}(x)\right)=v\left(k_{n+1}\right)\right\}}{\mathbf{P}\left\{v\left(a_{1}(x)\right)=v\left(k_{1}\right), \ldots, v\left(a_{n}(x)\right)=v\left(k_{n}\right)\right\}} \\
& =\frac{(p-1)^{n+1} p^{-\sum_{j=1}^{n} v\left(k_{j}\right)+v\left(k_{n+1}\right)} p^{-\sum_{j=1}^{n}\left(v\left(r_{j}\left(k_{j}\right)\right)-v\left(s_{j}\left(k_{j}\right)\right)\right)}}{(p-1)^{n} p^{-\sum_{j=1}^{n-1} v\left(k_{j}\right)+v\left(k_{n}\right)} p^{-\sum_{j=1}^{n-1}\left(v\left(r_{j}\left(k_{j}\right)\right)-v\left(s_{j}\left(k_{j}\right)\right)\right)}} \\
& =(p-1) p^{v\left(k_{n+1}\right)-2 v\left(k_{n}\right)-v\left(r_{n}\left(k_{n}\right)\right)+v\left(s_{n}\left(k_{n}\right)\right)} .
\end{aligned}
$$

On the other hand, write

$$
\begin{aligned}
& A_{n}=\left\{v\left(a_{n}(x)\right)=v\left(k_{n}\right), v\left(a_{n+1}(x)\right)=v\left(k_{n+1}\right)\right\}, \\
& B_{n}=\left\{v\left(a_{n}(x)\right)=v\left(k_{n}\right)\right\} .
\end{aligned}
$$

Also by Lemma 3.3, we have

$$
\begin{aligned}
\mathbf{P}\left\{v\left(a_{n+1}(x)\right)=v\left(k_{n+1}\right) \mid v\left(a_{n}(x)\right)=v\left(k_{n}\right)\right\} \\
\quad=\frac{\mathbf{P}\left\{v\left(a_{n}(x)\right)=v\left(k_{n}\right), v\left(a_{n+1}(x)\right)=v\left(k_{n+1}\right)\right\}}{\mathbf{P}\left\{v\left(a_{n}(x)\right)=v\left(k_{n}\right)\right\}} \\
\quad=\frac{\sum \mathbf{P}\left(\left\{v\left(a_{j}(x)\right)=v\left(l_{j}\right), 1 \leq j \leq n-1\right\} \cap A_{n}\right)}{\sum \mathbf{P}\left(\left\{v\left(a_{j}(x)\right)=v\left(m_{j}\right), 1 \leq j \leq n-1\right\} \cap B_{n}\right)} \\
=\frac{\sum p^{-\sum_{j=1}^{n-1} v\left(l_{j}\right)-v\left(k_{n}\right)} p^{-\sum_{j=1}^{n-1}\left(v\left(r_{j}\left(l_{j}\right)\right)-v\left(s_{j}\left(l_{j}\right)\right)\right)-\left(v\left(r_{n}\left(k_{n}\right)\right)-v\left(s_{n}\left(k_{n}\right)\right)\right)}}{(p-1)^{-1} \sum p^{-\sum_{j=1}^{n-1} v\left(m_{j}\right)+v\left(k_{n}\right)-v\left(k_{n+1}\right)} p^{-\sum_{j=1}^{n-1}\left(v\left(r_{j}\left(m_{j}\right)\right)-v\left(s_{j}\left(m_{j}\right)\right)\right)}} \\
=(p-1) p^{v\left(k_{n+1}\right)-2 v\left(k_{n}\right)-v\left(r_{n}\left(k_{n}\right)\right)+v\left(s_{n}\left(k_{n}\right)\right)},
\end{aligned}
$$

where the summations in the numerators of (11) and (12) are over all $l_{1}, \ldots, l_{n-1} \in S_{p}$ satisfying $v\left(l_{1}\right) \leq-1, v\left(l_{j+1}\right) \leq 2 v\left(l_{j}\right)-1+v\left(r_{j}\left(l_{j}\right)\right)-$ $v\left(s_{j}\left(l_{j}\right)\right)$ for $1 \leq j \leq n-2$ and $v\left(k_{n}\right) \leq 2 v\left(l_{n-1}\right)-1+v\left(r_{n-1}\left(l_{n-1}\right)\right)-$ $v\left(s_{n-1}\left(l_{n-1}\right)\right)$, and the summations in the denominators of (11) and (12) are over all $m_{1}, \ldots, m_{n-1} \in X_{p}$ satisfying $v\left(m_{1}\right) \leq-1, v\left(m_{j+1}\right) \leq 2 v\left(m_{j}\right)-$ $1+v\left(r_{j}\left(m_{j}\right)\right)-v\left(s_{j}\left(m_{j}\right)\right)$ for $1 \leq j \leq n-2$ and $v\left(k_{n}\right) \leq 2 v\left(m_{n-1}\right)-1+$ $v\left(r_{n-1}\left(m_{n-1}\right)\right)-v\left(s_{n-1}\left(m_{n-1}\right)\right)$.

From (2), for any $k, l \in S_{p}$ satisfying $v(k)=v(l) \leq-1$, we have

$$
v\left(r_{n}(k)\right)=v\left(r_{n}(l)\right), \quad v\left(s_{n}(k)\right)=v\left(s_{n}(l)\right),
$$


for any $n \geq 1$. From now on, for any $j \geq 1$, we write $v\left(r_{n}(k)\right)=r(n, j)$ and $v\left(s_{n}(k)\right)=s(n, j)$ if $k \in S_{p}$ with $v(k)=-j$.

For any $x \in X_{p}$, let $\left\{\triangle_{n}(x): n \geq 0\right\}$ be the sequence of random variables such that $\triangle_{0}(x)=v\left(a_{1}(x)\right)$ and $\triangle_{n}(x)=v\left(a_{n+1}(x)\right)-2 v\left(a_{n}(x)\right)-$ $v\left(r_{n}\left(a_{n}(x)\right)\right)+v\left(s_{n}\left(a_{n}(x)\right)\right)$ for $n \geq 1$.

Now we prove our key result.

Proposition 3.5. $\left\{\triangle_{n}(x): n \geq 0\right\}$ is a sequence of independent and identical distributed random variables, and for any $k \geq 1$,

$$
\mathbf{P}\left\{x \in S_{p}: \triangle_{n}(x)=-k\right\}=\frac{p-1}{p^{k}} .
$$

Proof. For any $n \geq 1$ and $k \geq 1$, by Proposition 3.4, $\mathbf{P}\left\{x \in X_{p}: \triangle_{n}(x)=-k\right\}$

$$
\begin{aligned}
= & \sum_{j=1}^{\infty} \mathbf{P}\left\{\triangle_{n}(x)=-k \mid v\left(a_{n}(x)\right)=-j\right\} \mathbf{P}\left\{v\left(a_{n}(x)\right)=-j\right\} \\
= & \sum_{j=1}^{\infty} \mathbf{P}\left\{v\left(a_{n+1}(x)\right)-2 v\left(a_{n}(x)\right)-v\left(r_{n}\left(a_{n}(x)\right)\right)\right. \\
& \left.\quad+v\left(s_{n}\left(a_{n}(x)\right)\right)=-k \mid v\left(a_{n}(x)\right)=-j\right\} \mathbf{P}\left\{v\left(a_{n}(x)\right)=-j\right\} \\
= & \sum_{j=1}^{\infty} \mathbf{P}\left\{v\left(a_{n+1}(x)\right)=-2 j-k+r(n, j)-s(n, j) \mid v\left(a_{n}(x)\right)=-j\right\} \\
= & \sum_{j=1}^{\infty} \mathbf{P}\left\{v\left(a_{n}(x)\right)=-j\right\} \cdot \frac{p-1}{p^{k}}=\frac{p-1}{p^{k}} .
\end{aligned}
$$

Also it is easy to see that for any $k \geq 1$,

$$
\mathbf{P}\left\{x \in X_{p}: \triangle_{0}(x)=-k\right\}=\frac{p-1}{p^{k}} .
$$

Now we prove that the random variables $\triangle_{n}(x), n=0,1, \ldots$, are independent. For any positive integers $k_{1}, \ldots, k_{n+1}$,

$$
\begin{aligned}
\mathbf{P}\left\{x \in X_{p}: \triangle_{0}(x)=-k_{1}, \triangle_{1}(x)=-k_{2}, \ldots, \triangle_{n}(x)=-k_{n+1}\right\} \\
\quad=\mathbf{P}\left\{x \in X_{p}: v\left(a_{1}(x)\right)=p_{1}, v\left(a_{2}(x)\right)=p_{2}, \ldots, v\left(a_{n+1}(x)\right)=p_{n+1}\right\},
\end{aligned}
$$

where $p_{1}, \ldots, p_{n+1}$ are defined as follows: $p_{1}=-k_{1}$, and for any $1 \leq j \leq n$,

$$
p_{j+1}=2 p_{j}-k_{j+1}+r\left(j,-p_{j}\right)-s\left(j,-p_{j}\right) .
$$

By the definition of $\left\{p_{j}: 1 \leq j \leq n+1\right\}$, we have

$$
p_{n+1}=\sum_{j=1}^{n} p_{j}-\sum_{j=1}^{n+1} k_{j}+\sum_{j=1}^{n}\left(r\left(j,-p_{j}\right)-s\left(j,-p_{j}\right)\right) .
$$


Thus by Lemma 3.3,

$$
\begin{aligned}
& \mathbf{P}\left\{\triangle_{0}(x)=-k_{1}, \triangle_{1}(x)=-k_{2}, \ldots, \triangle_{n}(x)=-k_{n+1}\right\} \\
& =(p-1)^{n+1} p^{-\sum_{j=1}^{n} p_{j}+p_{n+1}} p^{-\sum_{j=1}^{n}\left(r\left(j,-p_{j}\right)-s\left(j,-p_{j}\right)\right)}=(p-1)^{n+1} p^{-\sum_{j=1}^{n+1} k_{j}} \\
& =\mathbf{P}\left\{\triangle_{0}(x)=-k_{1}\right\} \mathbf{P}\left\{\triangle_{1}(x)=-k_{2}\right\} \ldots \mathbf{P}\left\{\triangle_{n}(x)=-k_{n+1}\right\} .
\end{aligned}
$$

LEMMA 3.6. For every $n \geq 0$, the random variable $\triangle_{n}(x)$ has mean value and variance

$$
\mathbf{E}\left(\triangle_{n}(x)\right)=-\frac{p}{p-1}, \quad \operatorname{Var}\left(\triangle_{n}(x)\right)=\frac{p}{(p-1)^{2}} .
$$

Proof. By Proposition 3.5,

$$
\mathbf{E}\left(\triangle_{n}(x)\right)=\sum_{k=1}^{\infty}-k \mathbf{P}\left\{\triangle_{n}(x)=k\right\}=\sum_{k=1}^{\infty}-k \cdot \frac{p-1}{p^{k}}=-\frac{p}{p-1} .
$$

Similarly,

$$
\mathbf{E}\left(\triangle_{n}(x)^{2}\right)=\sum_{k=1}^{\infty}(-k)^{2} \mathbf{P}\left\{\triangle_{n}(x)=-k\right\}=\sum_{k=1}^{\infty} k^{2} \cdot \frac{p-1}{p^{k}}=\frac{p}{p-1}+\frac{2 p}{(p-1)^{2}},
$$

thus

$$
\operatorname{Var}\left(\triangle_{n}(x)\right)=\mathbf{E}\left(\triangle_{n}(x)^{2}\right)-\left(\mathbf{E}\left(\triangle_{n}(x)\right)\right)^{2}=\frac{p}{(p-1)^{2}}
$$

Proof of Theorem 2.4. (i) By Proposition 3.5 and Lemma 3.6, $\left\{\triangle_{n}(x)\right.$ : $n \geq 0\}$ is a sequence of independent and identical distributed random variables with mean value $-p /(p-1)$ and variance $p /(p-1)^{2}$. Hence by the central limit theorem (see [1, p. 317, Corollary 2]), we have

$$
\lim _{n \rightarrow \infty} \mathbf{P}\left\{x \in X_{p}: \frac{\sum_{j=0}^{n-1} \triangle_{j}(x)+\frac{p}{p-1} n}{\sqrt{n p} /(p-1)}<t\right\}=\frac{1}{\sqrt{2 \pi}} \int_{-\infty}^{t} e^{-u^{2} / 2} d u
$$

and thus part (i) of Theorem 2.4 follows.

(ii) By the strong law of large numbers (see [1, p. 125, Corollary 2]), we have, for $\mathbf{P}$-almost all $x \in X_{p}$,

$$
\lim _{n \rightarrow \infty} \frac{1}{n} \sum_{j=0}^{n-1} \triangle_{j}(x)=-\frac{p}{p-1}
$$

and the proof of part (ii) of Theorem 2.4 is finished.

(iii) By the iterated logarithm law (see [1, p. 373, Theorem 2]), we have, for $\mathbf{P}$-almost all $x \in X_{p}$,

$$
\limsup _{n \rightarrow \infty} \frac{\sum_{j=0}^{n-1} \triangle_{j}(x)+\frac{p}{p-1} n}{\sqrt{2 n \log \log n}}=\frac{\sqrt{p}}{p-1},
$$




$$
\liminf _{n \rightarrow \infty} \frac{\sum_{j=0}^{n-1} \triangle_{j}(x)+\frac{p}{p-1} n}{\sqrt{2 n \log \log n}}=-\frac{\sqrt{p}}{p-1},
$$

and we finish the proof of part (iii).

We now list some special cases and give applications of Theorem 2.4 to these special expansions. The metric properties of $p$-adic Lüroth series expansions have been discussed in A. Knopfmacher and J. Knopfmacher [11], and Grabner and A. Knopfmacher [5] have investigated the corresponding results for $p$-adic Engel series expansions. It is easy to check that (1) holds in all of the following cases.

EXAmple 1. For any $a \in p^{-1}\left(S_{p} \backslash\{0\}\right)$ and any $n \geq 1$, let $s_{n}(a)=$ $a(a-1), r_{n}(a)=1$. Then the algorithm (3) leads the $p$-adic Lüroth series expansion of $x \in X_{p}$,

$$
x=\frac{1}{a_{1}(x)}+\sum_{n=2}^{\infty} \frac{1}{a_{1}(x)\left(a_{1}(x)-1\right) \ldots a_{n-1}(x)\left(a_{n-1}(x)-1\right) a_{n}(x)} .
$$

In this case, $\triangle_{n}(x)=v\left(a_{n+1}(x)\right)$ for any $n \geq 0$. By Theorem 2.4 , we have

Corollary 3.7 ([11]). For p-adic Lüroth series expansions:

(i) $\lim _{n \rightarrow \infty} \mathbf{P}\left\{x \in X_{p}: \frac{\sum_{j=0}^{n-1} v\left(a_{j}(x)\right)+\frac{p}{p-1} n}{\sqrt{n p} /(p-1)}<t\right\}=\frac{1}{\sqrt{2 \pi}} \int_{-\infty}^{t} e^{-u^{2} / 2} d u$.

(ii) For $\mathbf{P}$-almost all $x \in X_{p}$,

$$
\lim _{n \rightarrow \infty} \frac{1}{n} \sum_{j=0}^{n-1} v\left(a_{j}(x)\right)=-\frac{p}{p-1}
$$

(iii) For $\mathbf{P}$-almost all $x \in X_{p}$,

$$
\begin{aligned}
& \limsup _{n \rightarrow \infty} \frac{\sum_{j=0}^{n-1} v\left(a_{j}(x)\right)+\frac{p}{p-1} n}{\sqrt{2 n \log \log n}}=\frac{\sqrt{p}}{p-1}, \\
& \liminf _{n \rightarrow \infty} \frac{\sum_{j=0}^{n-1} v\left(a_{j}(x)\right)+\frac{p}{p-1} n}{\sqrt{2 n \log \log n}}=-\frac{\sqrt{p}}{p-1} .
\end{aligned}
$$

ExAmple 2. For any $a \in p^{-1}\left(S_{p} \backslash\{0\}\right)$ and any $n \geq 1$, let $s_{n}(a)=a$ and $r_{n}(a)=1$. Using the algorithm (3), we get the $p$-adic Engel series expansion of $x \in X_{p}$,

$$
x=\sum_{n=1}^{\infty} \frac{1}{a_{1}(x) \ldots a_{n}(x)} .
$$

Now $\triangle_{0}(x)=v\left(a_{1}(x)\right)$, and $\triangle_{n}(x)=v\left(a_{n+1}(x)\right)-v\left(a_{n}(x)\right)$ for any $n \geq 1$. By Theorem 2.4, we have 
COROllary 3.8 ([5]). For p-adic Engel series expansions:

(i) $\lim _{n \rightarrow \infty} \mathbf{P}\left\{x \in X_{p}: \frac{v\left(a_{n}(x)\right)+\frac{p}{p-1} n}{\sqrt{n p} /(p-1)}<t\right\}=\frac{1}{\sqrt{2 \pi}} \int_{-\infty}^{t} e^{-u^{2} / 2} d u$.

(ii) For $\mathbf{P}$-almost all $x \in X_{p}$,

$$
\lim _{n \rightarrow \infty} \frac{1}{n} v\left(a_{n}(x)\right)=-\frac{p}{p-1} .
$$

(iii) For $\mathbf{P}$-almost all $x \in X_{p}$,

$$
\begin{aligned}
& \limsup _{n \rightarrow \infty} \frac{v\left(a_{n}(x)\right)+\frac{p}{p-1} n}{\sqrt{2 n \log \log n}}=\frac{\sqrt{p}}{p-1}, \\
& \liminf _{n \rightarrow \infty} \frac{v\left(a_{n}(x)\right)+\frac{p}{p-1} n}{\sqrt{2 n \log \log n}}=-\frac{\sqrt{p}}{p-1} .
\end{aligned}
$$

EXAmple 3. For any $a \in p^{-1}\left(S_{p} \backslash\{0\}\right)$ and any $n \geq 1$, let $s_{n}(a)=1$ and $r_{n}(a)=1$ for all $n \geq 1$. The algorithm (3) yields the $p$-adic Sylvester series expansion of $x \in X_{p}$,

$$
x=\sum_{n=1}^{\infty} \frac{1}{a_{n}(x)} .
$$

Here $\triangle_{0}(x)=v\left(a_{1}(x)\right)$, and $\triangle_{n}(x)=v\left(a_{n+1}(x)\right)-2 v\left(a_{n}(x)\right)$ for any $n \geq 1$. From Theorem 2.4, we have

Corollary 3.9. For p-adic Sylvester series expansions:

(i) $\lim _{n \rightarrow \infty} \mathbf{P}\left\{x \in X_{p}: \frac{v\left(a_{n}(x)\right)-\sum_{j=1}^{n-1} v\left(a_{j}(x)\right)+\frac{p}{p-1} n}{\sqrt{n p} /(p-1)}<t\right\}$

$$
=\frac{1}{\sqrt{2 \pi}} \int_{-\infty}^{t} e^{-u^{2} / 2} d u \text {. }
$$

(ii) For $\mathbf{P}$-almost all $x \in X_{p}$,

$$
\lim _{n \rightarrow \infty} \frac{1}{n}\left(v\left(a_{n}(x)\right)-\sum_{j=1}^{n-1} v\left(a_{j}(x)\right)\right)=-\frac{p}{p-1} .
$$

(iii) For $\mathbf{P}$-almost all $x \in X_{p}$,

$$
\begin{aligned}
& \limsup _{n \rightarrow \infty} \frac{v\left(a_{n}(x)\right)-\sum_{j=1}^{n-1} v\left(a_{j}(x)\right)+\frac{p}{p-1} n}{\sqrt{2 n \log \log n}}=\frac{\sqrt{p}}{p-1}, \\
& \liminf _{n \rightarrow \infty} \frac{v\left(a_{n}(x)\right)-\sum_{j=1}^{n-1} v\left(a_{j}(x)\right)+\frac{p}{p-1} n}{\sqrt{2 n \log \log n}}=-\frac{\sqrt{p}}{p-1} .
\end{aligned}
$$

EXAMPLE 4 . Let $s_{n}(a)=a$ and $r_{n}(a)=a+1$ for any $a \in p^{-1}\left(S_{p} \backslash\{0\}\right)$ and any $n \geq 1$. By the algorithm (3), we get the $p$-adic Cantor infinite 
product of $x \in X_{p}$,

$$
1+x=\prod_{n=1}^{\infty}\left(1+\frac{1}{a_{n}(x)}\right) .
$$

Here $\triangle_{0}(x)=v\left(a_{1}(x)\right)$, and $\triangle_{n}(x)=v\left(a_{n+1}(x)\right)-2 v\left(a_{n}(x)\right)$ for any $n \geq 1$. From Theorem 2.4, we have

Corollary 3.10. For p-adic Cantor infinite products:

(i) $\lim _{n \rightarrow \infty} \mathbf{P}\left\{x \in X_{p}: \frac{v\left(a_{n}(x)\right)-\sum_{j=1}^{n-1} v\left(a_{j}(x)\right)+\frac{p}{p-1} n}{\sqrt{n p} /(p-1)}<t\right\}$

$$
=\frac{1}{\sqrt{2 \pi}} \int_{-\infty}^{t} e^{-u^{2} / 2} d u
$$

(ii) For $\mathbf{P}$-almost all $x \in X_{p}$,

$$
\lim _{n \rightarrow \infty} \frac{1}{n}\left(v\left(a_{n}(x)\right)-\sum_{j=1}^{n-1} v\left(a_{j}(x)\right)\right)=-\frac{p}{p-1} .
$$

(iii) For $\mathbf{P}$-almost all $x \in X_{p}$,

$$
\begin{aligned}
& \limsup _{n \rightarrow \infty} \frac{v\left(a_{n}(x)\right)-\sum_{j=1}^{n-1} v\left(a_{j}(x)\right)+\frac{p}{p-1} n}{\sqrt{2 n \log \log n}}=\frac{\sqrt{p}}{p-1}, \\
& \liminf _{n \rightarrow \infty} \frac{v\left(a_{n}(x)\right)-\sum_{j=1}^{n-1} v\left(a_{j}(x)\right)+\frac{p}{p-1} n}{\sqrt{2 n \log \log n}}=-\frac{\sqrt{p}}{p-1} .
\end{aligned}
$$

EXAmple 5. The $p$-adic modified Engel series expansion of $x \in X_{p}$ is obtained from the algorithm (3) by taking $s_{n}(a)=a-1$ and $r_{n}(a)=1$ for all $n \geq 1$ and all $a \in p^{-1}\left(S_{p} \backslash\{0\}\right)$,

$$
x=\sum_{n=1}^{\infty} \frac{1}{\left(a_{1}(x)-1\right) \ldots\left(a_{n-1}(x)-1\right) a_{n}(x)} .
$$

For this expansion, $\triangle_{0}(x)=v\left(a_{1}(x)\right)$, and $\triangle_{n}(x)=v\left(a_{n+1}(x)\right)-v\left(a_{n}(x)\right)$ for any $n \geq 1$. By Theorem 2.4, we have

Corollary 3.11. For p-adic modified Engel series expansions:

(i) $\lim _{n \rightarrow \infty} \mathbf{P}\left\{x \in X_{p}: \frac{v\left(a_{n}(x)\right)+\frac{p}{p-1} n}{\sqrt{n p} /(p-1)}<t\right\}=\frac{1}{\sqrt{2 \pi}} \int_{-\infty}^{t} e^{-u^{2} / 2} d u$.

(ii) For $\mathbf{P}$-almost all $x \in X_{p}$,

$$
\lim _{n \rightarrow \infty} \frac{1}{n} v\left(a_{n}(x)\right)=-\frac{p}{p-1} .
$$


(iii) For $\mathbf{P}$-almost all $x \in X_{p}$,

$$
\begin{aligned}
& \limsup _{n \rightarrow \infty} \frac{v\left(a_{n}(x)\right)+\frac{p}{p-1} n}{\sqrt{2 n \log \log n}}=\frac{\sqrt{p}}{p-1}, \\
& \liminf _{n \rightarrow \infty} \frac{v\left(a_{n}(x)\right)+\frac{p}{p-1} n}{\sqrt{2 n \log \log n}}=-\frac{\sqrt{p}}{p-1} .
\end{aligned}
$$

4. Proof of Theorem 2.5. In this section, we use Proposition 3.5 and the central idea in the proof of Theorem 5 in [11] to prove Theorem 2.5.

Proof of Theorem 2.5. By Proposition 3.5, the random variables

$$
\left|\frac{a_{k+1}(x) s_{k}\left(a_{k}(x)\right)}{a_{k}(x)^{2} r_{k}\left(a_{k}(x)\right)}\right|_{p}=p^{-\triangle_{k}(x)}, \quad k=1,2, \ldots,
$$

are independent and identically distributed, and it is easy to check that $p^{-\triangle_{k}(x)}$ has infinite expectation. For any $k \leq n$, define

$$
\begin{aligned}
& U_{k}(x)=\left|\frac{a_{k+1}(x) s_{k}\left(a_{k}(x)\right)}{a_{k}(x)^{2} r_{k}\left(a_{k}(x)\right)}\right|_{p}, V_{k}(x)=0 \\
& \text { if }\left|\frac{a_{k+1}(x) s_{k}\left(a_{k}(x)\right)}{a_{k}(x)^{2} r_{k}\left(a_{k}(x)\right)}\right|_{p} \leq n \log _{p} n, \\
& V_{k}(x)=\left|\frac{a_{k+1}(x) s_{k}\left(a_{k}(x)\right)}{a_{k}(x)^{2} r_{k}\left(a_{k}(x)\right)}\right|_{p}, U_{k}(x)=0 \\
& \text { if }\left|\frac{a_{k+1}(x) s_{k}\left(a_{k}(x)\right)}{a_{k}(x)^{2} r_{k}\left(a_{k}(x)\right)}\right|_{p}>n \log _{q} n .
\end{aligned}
$$

Then

$$
\begin{aligned}
\mathbf{P}\{x & \left.\in X_{p}:\left.\left|\frac{1}{n \log _{q} n} \sum_{j=1}^{n}\right| \frac{a_{j+1}(x) s_{j}\left(a_{j}(x)\right)}{a_{j}(x)^{2} r_{j}\left(a_{j}(x)\right)}\right|_{p}-(p-1) \mid>\varepsilon\right\} \\
\leq & \mathbf{P}\left\{x \in X_{p}:\left|U_{1}(x)+\ldots+U_{n}(x)-(p-1) n \log _{p} n\right|>\varepsilon n \log _{p} n\right\} \\
& +\mathbf{P}\left\{x \in X_{p}: V_{1}(x)+\ldots+V_{n}(x) \neq 0\right\} .
\end{aligned}
$$

By Proposition 3.5,

$$
\begin{aligned}
\mathbf{P}\left\{x \in X_{p}: V_{1}(x)+\ldots\right. & \left.+V_{n}(x) \neq 0\right\} \\
& \leq n \mathbf{P}\left\{x \in X_{p}:\left|\frac{a_{2}(x) s_{1}\left(a_{1}(x)\right)}{a_{1}(x)^{2} r_{1}\left(a_{1}(x)\right)}\right|_{p}>n \log _{p} n\right\} \\
& =n \sum_{k: p^{k}>n \log _{p} n}(p-1) p^{-k} \leq \frac{p}{\log _{p} n}=o(1) .
\end{aligned}
$$


Also by Proposition 3.5, we have

$$
\begin{aligned}
\mathbf{E}\left(U_{1}(x)+\ldots+U_{n}(x)\right) & =n \mathbf{E}\left(U_{1}(x)\right), \\
\operatorname{Var}\left(U_{1}(x)+\ldots+U_{n}(x)\right) & =n \operatorname{Var}\left(U_{1}(x)\right),
\end{aligned}
$$

where

$$
\begin{aligned}
& \mathbf{E}\left(U_{1}(x)\right)=\sum_{p^{k} \leq n \log _{q} n} p^{k} \mathbf{P}\left(\triangle_{1}(x)=-k\right) \\
&=\sum_{p^{k} \leq n \log _{p} n} p^{-k}(p-1) p^{k}=(p-1) \log _{p}\left(\left[n \log _{p} n\right]\right), \\
& \operatorname{Var}\left(U_{1}(x)\right) \leq \mathbf{E}\left(U_{1}(x)^{2}\right)=\sum_{p^{k} \leq n \log _{p} n}(p-1) p^{k}<p n \log _{p} n .
\end{aligned}
$$

Chebyshev's inequality then yields

$$
\begin{aligned}
\mathbf{P}\left\{x \in X_{p}:\left|U_{1}(x)+\ldots+U_{n}(x)-n \mathbf{E}\left(U_{1}(x)\right)\right|>\varepsilon n \mathbf{E}\left(U_{1}(x)\right)\right\} & \\
\leq & \frac{n \mathbf{V a r}\left(U_{1}(x)\right)}{\left(\varepsilon n \mathbf{E}\left(U_{1}(x)\right)\right)^{2}}<\frac{p n^{2} \log _{p} n}{\left(\varepsilon(p-1) n \log \left(\left[n \log _{p} n\right]\right)\right)^{2}}=o(1) .
\end{aligned}
$$

Since $\mathbf{E}\left(U_{1}(x)\right) \sim(p-1) \log _{p} n$ as $n \rightarrow \infty$, Theorem 2.5 follows.

Acknowledgements. The author thanks the referee for his (her) valuable suggestions. This work was done during the author's visits to the LAMFA, CNRS 2270, Amiens. He would like to thank this institution for the warm hospitality.

\section{References}

[1] Y. S. Chow and H. Teicher, Probability Theory. Independence, Interchangeability, Martingales, 3rd ed., Springer Texts Statist., Springer, New York, 1997.

[2] P. Erdős, A. Rényi and P. Szüsz, On Engel's and Sylvester's series, Ann. Univ. Sci. Budapest. Eötvös Sect. Math. 1 (1958), 7-32.

[3] A. H. Fan and J. Wu, Metric properties and exceptional sets of the Oppenheim expansions over the field of Laurent series, prépublication de LAMFA, Université de Picardie, Amiens, no. 18, 2002.

[4] J. Galambos, Reprentations of Real Numbers by Infinite Series, Lecture Notes in Math. 502, Springer, Berlin, 1976.

[5] P. J. Grabner and A. Knopfmacher, Arithmetical and metric properties of p-adic Engel series expansions, Publ. Math. Debrecen, to appear.

[6] H. Jager and C. de Vroedt, Lüroth series and their ergodic properties, Nederl. Akad. Wetensch. Proc. Ser. A 72 (1969), 31-42.

[7] A. Ya. Khintchine [A. Ya. Khinchin], Continued Fractions, Noordhoff, Groningen, 1963.

[8] A. Knopfmacher and J. Knopfmacher, A product expansion in p-adic and other non-Archimedean fields, Proc. Amer. Math. Soc. 104 (1988), 1031-1035. 
[9] A. Knopfmacher and J. Knopfmacher, Series expansions in p-adic and other nonArchimedean fields, J. Number Theory 32 (1989), 297-306.

[10] —, - Infinite series expansions for p-adic numbers, ibid. 41 (1992), 131-145.

[11] - - - Metric properties of some special p-adic series expansions, Acta Arith. 76 (1996), 11-19.

[12] N. Koblitz, p-adic Numbers, p-adic Analysis, and Zeta-Functions, 2nd ed., Springer, 1984.

[13] V. Laohakosol, A characterization of p-adic Ruban continued fractions, J. Austral. Math. Soc. A 39 (1985), 300-305.

[14] V. Laohakosol and P. Ubolsri, p-adic continued fractions of Liouville type, Proc. Amer. Math. Soc. 101 (1987), 403-410.

[15] K. Mahler, Zur Approximation p-adischer Irrationalzahlen, Nieuw Arch. Wisk. 18 (1934), 22-34.

[16] A. Rényi, On Cantor's product, Colloq. Math. 6 (1958), 135-139.

[17] A. A. Ruban, Some metric properies of p-adic numbers, Siberian Math. J. 11 (1970), 176-180.

[18] T. Salát, Zur metrischen Theorie der Lürothschen Entwicklungen der reellen Zahlen, Czechoslovak Math. J. 18 (1968), 489-522.

[19] W. H. Schikhof, Ultrametric Calculus, Cambridge Univ. Press, 1984.

[20] F. Schweiger, Ergodic Theory of Fibred Systems and Metric Number Theory, Oxford Univ. Press, New York, 1995.

[21] V. G. Sprindžuk, Mahler's Problem in Metric Number Theory, Amer. Math. Soc., 1969.

Department of Mathematics

Wuhan University

Wuhan, Hubei, 430072, P.R. China

E-mail: wujunyu@public.wh.hb.cn

Received on 3.6.2002

and in revised form on 20.8.2003 